\title{
A spatial analysis of the JBA headquarters in Splinter Cell: Double Agent.
}

\begin{abstract}
Three measures drawn from space syntax are proposed as means of describing game spaces with a view to a morphological critical analysis. These measures are: core integration and segregation, isovist area, and visibility/accessibility discrepancy (VAD). The measures are applied to the JBA headquarters levels in the stealth game Splinter Cell: Double Agent in order to evaluate the space in terms of the navigational challenges and affordances with which it presents the player and in terms of the rhythms of tension and relief inherent in its layout. It is demonstrated how these challenges and rhythms are married to the specific game tasks and events that each level contains.
\end{abstract}

\section{KEYWORDS}

Space syntax, Splinter Cell, isovists, visual analysis

\section{INTRODUCTION}

One important aspect of many videogames is the morphology of the game space. The layout of a game space can be an important factor in determining the level and type of challenge the game presents, the strategic and tactical options made available to the player, and the level's atmosphere, or how it feels to inhabit the space. It is useful, therefore, to develop a critical language that is able to describe different game morphologies with some precision.

While some writing on games has been content to talk about game space using general terms such as 'open' and 'linear,' there have been efforts made to treat the topic with more analytical rigour.

Ernest Adams examines architecture in games, by which he means both buildings and landscape, and suggests it serves primary and secondary functions [1]. The primary functions are to do with gameplay and include constraint, concealment, obstacles and exploration. The 
secondary functions are to establish the game world, give it a certain atmosphere and provide the player with information. Michael Nitsche identifies several kinds of spatial structures that we find in games [22]. These are tracks and rails, labyrinths and mazes, and arenas. By putting these forms together in different ways designers control the kind of experience that a level offers players.

Steven Chen and Duncan Brown adopt a similarly architectural approach to level design [5]. Drawing on Francis Ching's introductory architectural textbook they look at game space in terms of circulation patterns and overall organization of the space. Kenneth Hullett and Jim Whitehead have taken a more focused analytical look at design patterns in single player first person shooters [16]. They identify nine recurring patterns in the genre, such as sniper positions and choke points, describing them and detailing their impact on the game.

While these writers have correctly identified the relationship between form and experience in videogame space, we do not have a method for looking in detail at the configurations that underpin this relationship. The tendency is to look at broad types of spaces: arenas, sniper points etc. rather than the local spatial relationships that give rise to these types. In order to establish an approach to the morphology of game space that can conduct a critical analysis at this more abstract level, an adaptation of the architectural and urban planning theory of space syntax is proposed.

\section{SPACE SYNTAX}

First described in Bill Hillier and Julienne Hanson's The Social Logic of Space, space syntax is a method of investigating the internal relationships of a spatial system [14]. Just as linguistic syntax refers not to individual units of speech but to the way in which these units are arranged into sentences, so space syntax focuses on the arrangement of discrete spaces in a larger system. This goes beyond looking at the relationship of contiguous spaces. Knocking through a wall to connect neighbouring rooms in a house clearly affects the character of those rooms, but it also affects the house as a whole. Specifically, it will affect the way the house is used by its inhabitants, who may begin to prefer different routes and neglect routes that were previously preferred. This in turn alters the function and atmosphere of the rooms affected. Space syntax seeks to describe this more complex set of relationships. It is based on the insight that the character of a spatial system, whether a house, a museum, or a city district, is determined not 
only by its visual properties such as material, décor and architectural 'set-pieces' such as colonnades and architraves, but also, and often primarily, by the relationship of each space to every other space in the system. This set of relationships is known as configuration and is defined by Hillier as 'the simultaneously existing relations amongst the parts which make up the whole' [12].

While much architectural theory aims at the conscious level - those things we see and consciously grasp - space syntax looks at aspects of architecture that are intuitively felt, but not easily vocalised. They are, Hillier suggests, 'non-discursive,' working 'below the level of consciousness' [13]. But these relationships are, it is claimed, central to how space is used.

Different kinds of spatial structures lead to different patterns of engagement. Certain spatial formations rigorously determine how they will be used. The traditional layout of a church or a courtroom, for example, in which the entrances and exits of each user are prescribed and everyone is aware of where they can and cannot go, is of this kind. Others, such as a public park, allow for users to engage more freely, choosing their own routes through space. Hillier distinguishes between these two models of space, calling the first a long model and the second a short model. Long models tend toward conformity and conservation, whereas short models tend toward the inverse of these: individuation and morphogenesis [13]. In spatial terms, this means that long models tend to be similar to each other (conformity) and tend to reflect in their form the rules that constitute them (conservative), whereas short models tend to be more variable (individuation) and give rise to new spatial relationships (morphogenesis). However, this is not solely a predictor of spatial layout but also of the social relations that obtain through this layout. The space of religious ritual tends to strictly determine not only the relationship between spaces within the system but also between people who use those spaces. Buildings based on a long model, such as churches and courthouses, 'conserve given social statuses and relationships' whereas those based on a short model, such as parks and social clubs 'generate relations over and above those given by the social situation' [13: 6].

In an insight related to museum design, but that may be of particular relevance to videogame criticism, Sophia Psarra relates the long and short model to Umberto Eco's notion of the closed and open text. She suggests that 'the former prescribes experience, while the latter shapes a social pattern of social co-presence' [29: 230]. Psarra sees these two modes as embodying two different types of buildings, stable and variable: 
buildings in the first group are concerned with unambiguous semantic expression using the spatial properties to strengthen a representational geometric order and the content of the collection. The second group consists of works that are 'open' allowing spaces and artefacts to enter into multiple relationships rather than structuring interpretation [29: 230].

Psarra sees the Victorian museum, for example London's Natural History Museum and Chiswick Villa in the former category; the more post-modern NY MOMA and John Soame's house-museum she sees in the latter.

A similar distinction, though with different terminology, is made by Yoon Kyung Choi [7]. Here, focussing on the relationship between a building's configuration and its mode of interaction, the long model becomes 'the deterministic model' and the short model becomes the 'probabilistic model.' The former 'dictates viewing sequences and channels encounters in limited ways,' whereas the latter 'modulates exploration and encounter statistically according to the syntactic properties of the layout.' [7: 241] Choi gives the example of the Hammer Building in the Los Angeles County Museum of Art as a deterministic model, in which visitors simply move from gallery to gallery in a pre-determined sequence. It should be noted that the probabilistic model does not give rise to a simply random pattern of movement in the museum but rather movement that is a function of the way spaces are interconnected. In other words, it should be possible to predict within certain statistical parameters the kind of movement that the probabilistic model will give rise to. This idea of conservative and generative spaces has clear applications for providing a method of analysis that goes beyond terms like 'linear' and 'open' and instead looks at how movement through open worlds might be understood and structured in terms of the design of those worlds.

This approach to an analysis of videogames could be described in terms of formalist criticism. $20^{\text {th }}$ century schools of literary criticism like New Criticism and Russian Formalism emerged in reaction to psychological and historical methods to understand how literature worked in itself; how particular poems created specifically literary effects through identifiable and analysable literary techniques. This kind of criticism in game studies would look to the techniques through which games affect players. It would bracket off certain aspects of player 
personality and competence, generic conventions and designer intention in order to concentrate on these techniques in themselves. This is not to say that these other elements cannot be reintegrated with a formal analysis to develop a fuller picture of the experience of game-playing, but in order to identify the important formal elements they must first be described independently. This approach may be useful in describing how a space feels to play to a reader who is unfamiliar with the game. This is important in game studies since we are often writing about games that we cannot expect all of our readers to have played, much less to have played to completion.

Psarra looks at a number of public buildings, including several galleries, using space syntax to look at the relationship between architectural structure and perceptual and motor experiences. She attempts to bridge the gap between the space as conceptual and space as experiential, seeing space syntax as a method of analysing how the designed space relates to the observed or experienced space. This relationship between the form of the designed space and the experience it gives rise to is of clear importance in game environments.

Hillier claims that buildings transmit culture but 'invariably we handle domestic space patterns without thinking of them and even without being aware of them until they are challenged' [13: 30]. Perhaps this challenging of our unconsciousness of space is precisely what is happening in many games. Space is foregrounded in games in a way that language is foregrounded in poetry; forcing us to take stock of a taken-for-granted tool of cultural transmission. Hillier explains:

Architecture begins when the configurational aspects of form and space, through which buildings become cultural and social objects, are treated not as unconscious rules to be followed, but are raised to the level of conscious, comparative thought, and in this way made part of the object of creative attention [13:32].

In order to adapt space syntax to videogame analysis, this paper will offer a critical appraisal of one game using methods drawn from the discipline. Space syntax takes complex three-dimensional systems and models them in two dimensions. The model is then used to describe relationships of vision and access across the system through a series of measures. This 
paper will look at three measures using three different models of the JBA headquarters in Splinter Cell: Double Agent [33].

\subsection{The axial map}

Firstly, an axial map will be used to measure how the system's integration changes over the course of the game. Integration with the axial map is perhaps the most used measure in space syntax [25]. An axial map is a map of the system consisting of the fewest, longest lines of sight and movement needed to cover the entire system. Integration is a measure of the depth of each of these lines to every other line in the system. This depth is in terms of the number of lines between two spaces. That is, it is a measure of step distance rather than metric distance. In the simple corridor system in Figure 1, destinations A and B are about the same metric distance from the origin, but B is closer in terms of step distance. In large systems, it is possible to calculate integration for the system as a whole (global integration) or just counting lines within a certain radius (local integration).

\section{Figure 1: Example of simple axial map. $A$ and $B$ are metrically equidistant from the origin but $B$ is only two steps away whereas $A$ is four steps away.}

Integration has been found to be useful in two respects, what we might term the analytical and the predictive. Analytically, it has been found that sets of buildings with different topologies tend to assign similar social functions to rooms with similar integration values. This has been found in homes [15] and in factories [26]. In terms of prediction, integration has been found to be predictive of aggregate movement, with integrated routes preferred to segregated routes during search tasks [27]. Some research has been done on space syntax measures as predictive of movement through virtual environments. Ruth Conroy's $2001 \mathrm{PhD}$ thesis explores spatial navigation using a head-mounted display and finds similar results to those found in real world environments, particularly in relation to a measure derived from integration, intelligibility [9]. Conroy finds that intelligibility is predictive of direct movement between origin and destination, no redundancy, similar routes taken by different participants, and pauses at major decision points. In unintelligible environments routes were more distributed, indirect and inefficient, with 
more randomly located pause points and less consistency between participants. Other research has found correlation between movement in computer-mediated and non-mediated environments, for example in a museum [11] and an airport [24].

Little research exists in predicting movement patterns in games. In O Cho and Young Ook Kim have analysed player movement patterns in World of Warcraft from a space syntax perspective [6]. They found that global, but not local, integration is highly correlated with player movement. This is slightly surprising since local integration is usually a good predictor of pedestrian traffic in real world environments. They suggest that this is due to a fundamental difference in how people navigate in the real world and the game world, partly due to the ready availability of an in-game map. Players tend to use their map to plan the route to their target destination before they set out, whereas people navigating in real environments tend to adapt their route as they move based on their changing perceptions. This is an example of the necessity of taking into account the differences between real world and game navigation in understanding game spaces.

Richard Michael Levy and Mary Grantham O’Brien use an axial map in order to predict player movement in a game designed to teach the German language to high school students [20] . Unfortunately, the paper does not publish the results based on local or global integration, which are the measures most useful in predicting movement. However, they do claim that integration correlates with paths taken by the students.

Of these two uses of space syntax - the analytical and the predictive - the present study falls into the former type. The main focus is on whether integration can be useful in thinking about how configuration establishes the character and role of particular spaces in the system rather than whether we can use it to predict player behaviour. However, the latter, predictive function of space syntax has potential applications in designing open and complex game spaces that encourage certain patterns of movement. Further empirical work would be necessary to demonstrate its usefulness in this regard.

\subsection{Visual graph analysis (VGA)}

The second measure used in this paper involves visual graph analysis (VGA). VGA proceeds by laying a grid over the plan of the space and then calculating the connections from the centre of each square to every other square. This represents what can be seen from each point 
in the space. From this we can calculate various measures, including isovist properties throughout the system. An isovist is a polygon that represents the total visibility field from a given point [2]. Figure 2 shows the same corridor system as before, this time with isovists from point $\mathrm{A}$ and the origin represented.

Figure 2: Example of same corridor showing two isovists; the isovist from the origin, in red, has a relatively large area, while the isovist from point $A$, in blue, has a relatively small area.

Each isovist has certain properties which can be used to describe its visual field. Using Depthmap, a piece of VGA software developed at UCL, we can display this information for all isovists across the level or across a section of the level [32]. The present paper will look at one isovist property: the overall area. This measure gives an idea of the visibility of each space and also how much of the complex can be seen from each space.

Incidentally, it is also possible to calculate integration on the visual graph in much the same way as it is calculated on the axial map. Here, the visual steps from each grid square to every other grid square are calculated. That is, the grid square rather than the axial line is used as the base unit.

\subsection{Visibility/Accessibility Discrepancy (VAD)}

Lastly, a new measure will be introduced to take account of the discrepancy between visibility and accessibility that is integral to the game. This discrepancy as it exists in the real world has been explored from the perspective of space syntax by Daniel Koch, for whom it is a key element of architecture [18]. For him, ‘[a]rchitecture, and especially in the modern sense, emerges when Visibility and Accessibility are disconnected' and this disconnection is 'one of the most pervasive, effective, and powerful means through which architecture formulates social significance and social meaning' [18: np]. This has clear applications in the world of videogames, where a frequent question is: 'I can see where I want to go but how do I get there?' In these sorts of games lines of sight are simple, lines of access are complex. It is this obviousness by sight but difficulty by movement that creates the delight of puzzle games such as Braid, Limbo and Portal [23, 28, 34]. 
A key term in space syntax as it is practiced in relation to real environments is 'social.' The central argument in The Social Logic of Space [14] is that space constitutes the social and that particular spatial configurations are both determined by existing social relationships and help to maintain and illustrate those relationships. Particular spatial configurations may also help to generate new social relationships. In certain kinds of games, especially massively multiplayer online games it may be fruitful to maintain this emphasis on the social. But another approach, and the one adopted here, is to substitute the notion of gameplay for the social. The key relationship is not between people but between the player and game goals, tasks and objects.

For example, I have suggested that puzzle games make use of the discrepancy between visibility and accessibility. However, when I am staring at the screen in Braid wondering how to get to the next platform that seems 'so near yet so far,' the frustrations that the visibility/accessibility discrepancy gives rise to do not shape my relationship to other people but my relationship to the game.

Koch identifies several architectural figures in which accessibility and visibility are differentiated in a way that gives rise to meaning, which he defines as a description of 'identities, roles and social relations' [18: np]. For Koch, the social involves the way in which space mediates encounter and avoidance. But while encounter and avoidance are the key ways that the social and the spatial interact in the real world, encounter and avoidance in games, while still being mediated by spatial configuration, may relate less to social identity but will necessarily relate to the kind of gameplay that the game puts forward. The same figures, therefore, when encountered in games can give rise to a different kind of meaning; one that is descriptive and generative of certain kinds of gameplay rather than certain kinds of social identity.

Koch's architectural figures include the balcony, the catwalk and the glass box. The balcony 'allows someone to see something that is comparatively far away in terms of access' [18: np]. The catwalk has the same distinction, but instead of the many below being on view to the few in the balcony, here the few on the catwalk are on view to the many below. Context and conventions can determine whether the stage in question is a balcony or a catwalk, but there do seem to be some spatial elements that influence whether a stage is more likely to be one or the other. These ensure that the occupants of the balcony are relatively hidden from the public gaze and the occupants of the catwalk are relatively exposed to it. This may be accomplished by the arrangement of the lower floor either in such a way as to direct attention to or away from the 
stage. Balconies will often have a railing or half wall that obstructs part of the viewer's body from the people below, whereas the catwalk requires its occupant to be fully visible. Balconies usually allow the viewer to move quickly to a more secluded, private area, whereas catwalks thrust out amongst the viewing public, not only preventing an easy escape from the glare but also allowing the occupant to be viewed from several angles at once. In games, the sniper spot is perhaps the most obvious example of the balcony/catwalk. A sniper spot that is too much of a balcony will likely see players camp out for entire games picking off easy targets. If it is too much of a catwalk, the sniper will be exposed before the chance to despatch any enemies arises. Take the example of this lookout on the Afghan multiplayer map of Call of Duty: Modern Warfare 2 [17].

Figure 3: Afghan map from Call of Duty: Modern Warfare 2. The black square box shows a possible sniper position.

In Figure 3 the black box shows a position from which a sniper can command a view of the central area around the crashed plane. To the east there is a sharp drop allowing for a wide view and preventing other players from approaching. The change in elevation neutralises much of the cover in the central area. The position slopes down to the west, allowing the sniper to easily retreat out of the visible range of players to the north and east. To this extent, it is a balcony, commanding a view of other players but not drawing attention to itself as a spectacle. However, from the west the position is more like a catwalk. The hill slopes gently down to the area marked 'air defense west,' providing an approach that allows other players to come close to the sniper. This area also provides plenty of cover behind barrels and walls. Contrastingly, there is no westward cover in the immediate vicinity of the sniper position, meaning that the sniper is at a disadvantage in a gunfight instigated from the west and is visible to players as soon as they enter the air defense west area, either from the south or the north.

However, if the sniper drops down to a ledge immediately below this point a position is found that has most of the benefits of the balcony and few of the weaknesses of the catwalk. Now the sniper cannot be seen by players entering the air defense west, decreasing the chances of a player stumbling upon the sniper at work. The sniper will only be discovered if a player 
seeks him out. Also, because the area is off the beaten track, it may be more difficult for a player in the plane area to locate the position of a sniper on this ledge. As players become more familiar with the maps these kinds of hiding spots become less powerful, but they remain an important element in rewarding exploration of the territory.

A third figure that Koch [18] describes is the glass box. Here, Koch is referring to the display cabinets used to house expensive and exclusive cosmetics brands and jewellery. In this instance, what seems to be close is in real terms far away. The contents are made tantalising by their proximity, but they retain an air of exclusivity and unattainability, giving rise to desire, by their distance. Glass boxes exist throughout games. An obvious example in first person shooters is the kill-cam. In recent Call of Duty games the kill-cam is triggered when a player dies, replaying the victim's death from the opponent's viewpoint. The kill-cam serves several functions. For example, it prevents camping by giving away the killer's position to the victim. But it also operates as a glass box. When the victim is temporarily shifted into the position of the killer, this allows a glance at the weaponry and perks that the killer has. For a moment, the victim sees the goodies that are attainable, indeed almost possesses them. But this only lasts as long as the kill-cam, after which the player is returned to the fight as before and forced to work toward attaining the previously glimpsed weapons. An extension of this is included from Modern Warfare 2, where a player can choose a perk that allows the player to steal some of the killer's properties on death.

Several writers and designers have applied 'weenies', a concept drawn from theme park construction, particularly Disneyland, to the design of game environments $[30,8,19]$. These are large landmarks that can be seen from a distance - such as Sleeping Beauty's castle in Disneyland - that draw guests' attention, help guests orient themselves, and give them cues for navigation. VAD offers a way of describing the relationship with some precision.

VAD is designed to measure the discrepancy between visibility and accessibility described by Koch. It is based on the convex map, which is a representation of the system as a set of convex spaces. A convex space is a polygon in which a straight line can be drawn between every point and every other point it contains. Convex maps are used to model co-presence in the system as each person in a convex space can see everyone else in that space.

VAD is measured by calculating the depth of a space measured in terms of access from the spaces with which it has a direct visual connection. This is the 'real depth' between two 
visually contiguous spaces. The VAD value is only calculated for spaces which have some discrepancy. In the example in Figure 4, room A is visually directly connected to the atrium and the stairs below because of the window but separated from them in terms of access. It therefore has a high VAD value, represented in red $^{1}$. The next highest value is for the atrium, since it is visually connected to only one distant space, room A. The stairs is also visually connected to room A, but since $\mathrm{A}$ is closer to the stairs ( 3 steps) than it is to the atrium (4 steps) the stairs has a lower value than the atrium. The visual connections from rooms $\mathrm{B}$ and $\mathrm{C}$ do not reach beyond their accessible connections and therefore they do not have an applicable VAD value and are given a value of zero.

\section{Figure 4: Example plan of two floors with window connecting atrium and room $A$, which are separated in terms of access by 4 spaces.}

We can expect a space that is a good surveillance spot to have direct visual connections to many spaces that are far away. But VAD can also identify areas which are vulnerable to surveillance and areas which may be landmarks that act as attractors. In the first case - the sniper spot - the high VAD area is a balcony, in the second - the area vulnerable to surveillance - it is a catwalk and in the third - the attractor - it is a glass box. We must look at contextual factors such as relative elevation, player position, lighting, and game and task type to differentiate between these three figures.

\section{Splinter Cell: Double Agent}

In Splinter Cell: Double Agent the player takes on the role of Sam Fisher, a National Security Agency (NSA) operative who has infiltrated a terrorist organization named John Brown's Army (JBA). This analysis will focus on three of the levels set in in the JBA headquarters (Figure 5). In each of these levels Sam has a particular set of objectives from the NSA and from JBA and is given a limited time in which to complete them. Completing tasks for JBA increases Sam's trust level with the terrorists, but may decrease his NSA trust level; completing tasks for the NSA may have the reverse effect. If too much trust is lost with either

\footnotetext{
${ }^{1}$ Throughout the paper warmer colours represent high values and cooler colours low values, regardless of the measure being discussed.
} 
party, the game is over. In each level, Sam gains access to parts of the complex previously off limits. At the same time, some parts of the complex become closed off. The purpose of this analysis is to consider the relationship between the changing configuration of the headquarters as understood through syntactical measures and the character of the different areas of the complex.

\section{Figure 5: Plan of the JBA headquarters.}

\subsection{Axial map integration}

\subsubsection{The first level}

In the first level set in the complex, JBA assigns Sam tasks in the training course area, located just off the long corridor in the main area. The primary NSA tasks are located in the server room and on the rooftop, with secondary tasks in the back room of the infirmary. Only about half of the total complex is accessible at this stage. Of these, the three areas necessary to the NSA missions are restricted, and if Sam is caught there JBA will lose trust in him.

\section{Figure 6: First level. The integration core is in red, and the segregation core is in} blue.

Figure 6 shows the axial map reduced to the 10\% most integrated lines (the integration core) and the $10 \%$ least integrated lines (the segregation core). The first observation to make is that the integration core corresponds closely to the busiest area in terms of non-player character (NPC) movement. Similarly, the segregation core is found in quieter more isolated areas. This correspondence helps the player believe in the complex as a real building. The NPCs are following scripted patrols but these patrols seem to be acting in line with what we would expect from this building. Highly integrated areas attract movement; segregated areas tend to be quieter.

Let us look at the three points of interface between the open and restricted areas. These are marked in Figure 6 as 1) at the rooftop, 2) between the infirmary and the infirmary's back office and 3) at the server room door. These are the three points where Sam must move between open and restricted areas and so are particularly conducive to tension. 'Tension' here is used to describe a form of aesthetic response that works in relation to relaxation or relief. Musical 
tension, for example, holds the listener in suspenseful anticipation of the resolution of a dissonant passage [3]. The parallel in a game occurs when a particular game state is uncomfortable but is capable of being resolved through a change in the game state brought about either through player or non-player action. In either case the aesthetic response is derived from the player's anticipation of this resolution. These liminal points between open and restricted areas are sources of tension because terrorist guards become suspicious when Sam spends time there, and Sam generally must perform a hack or pick manoeuvre to gain access. Their tension is derived from the fact that they place Sam in a vulnerable position - conducive to tension - in relation to a more safe position - conducive to relaxation or relief - on either side of the threshold. It may seem strange to say that being in the restricted area, where Sam is vulnerable, is a resolution of the tension of gaining access to the restricted area. This is the case because once Sam enters the restricted area it is possible to seek cover. While attempting to access the area such cover is impossible. One of these points of tension, the infirmary, is located on the integration core. The other two are one and two steps away from it. This difference in proximity to the integration core has a major impact on how anxious it feels trying to cross the threshold without being observed. Tension is highest when Sam must perform stealth actions in the busy integration core in trying to access the infirmary.

The integration core is a single unit of interconnected lines. This firmly establishes the area around the long corridor in the general quarters as the complex's hub. This is an essential means by which the player is orientated. Sam initially enters the complex by way of the north entrance to the main area on the ground floor, which offers a view of much of the ground floor and the TV area on the floor below (see Figure 7). Each level begins with Sam being led on a different trajectory through this area by one of the JBA members. The area is therefore not only central in terms of its configuration but also in how it is presented.

\section{Figure 7: Sam and the player's first impression of the JBA complex.}

One of the primary NSA mission is in the server room, which is in the segregation core. The placement of a mission in the relatively deep area of the segregation core provides the player with a challenge given the time constraint of the level. The structure of the server room mission 
involves two different kinds of challenges. There are two navigation style challenges - getting to and from the mission location - on either side of a more stationary challenge - this time planting a bug in the organization's server. This is a recurrent mission structure in games, and one found many times in the JBA headquarters levels of Splinter Cell. By placing the mission in a segregated area of the building, the game emphasizes the navigational aspect of the task.

\subsubsection{The second level}

In the second level more of the complex has opened up, and now Sam can enter the areas around the low security corridor (LSC) as well as the courtyard and the docks. The surveillance area is still inaccessible, and the access to the rooftop from the first level has been blocked off. During this level, the JBA missions are located in the mine assembly area, the furnace room and the training course. The main NSA mission is in Emile's quarters. There are also secondary missions in the officers' quarters and in Enrica's workshop.

\section{Figure 8: Second level integration and segregation core.}

As seen in Figure 8, the integration core has now shifted away from the main corridor to straddle the open access and restricted access areas at a different point. This is between the open mine assembly area and the restricted low security corridor (LSC) and the passages in between. This shift eastward is due to the opening up of a door in the mine assembly area and reflects an increase in the importance of this space, which is the site of the main JBA mission Sam is tasked with. This also brings the integration core to the door of the restricted server room. However, this area is unused in this level. As noted above, the previous level has the main interfaces between the open and restricted area just off the integration core. The shifting of the integration core brings it onto the main interface between the open and restricted areas. The balance between tension due to the 'busy' integration core and relief due to the relative isolation outside it is maintained in this level. 
The main NSA mission is located in Emile's office, which is near the segregation core. This is still in a fairly deep part of the complex, putting the same kind of time pressure on the player as was evident with the server room mission in the first level.

\subsubsection{The third level}

In the third level, the docks access has been closed off and the roof is still inaccessible, but Sam is now able to get through to the surveillance area. The primary JBA mission is located

in the common area with secondary tasks in the training course and the mine assembly area. The primary NSA mission is in the surveillance room, with a secondary mission in Enrica's workshop.

\section{Figure 9: Third level integration and segregation core.}

Figure 9 shows that the integration core shifts back to the west in this level due to the opening up of the door to the north of the LSC. This gives a direct point of contact between the main open access area and the main restricted area around the LSC without the intervening passages we get through the mine assembly area. As was the case with the location of the infirmary in the integration core in the first level, there is now a greater sense of tension when Sam is moving between the two zones. This is somewhat tempered by the fact that the north door to the low security corridor is on a dog leg and so is hidden from the view of the general quarters, but the difference between entering the restricted zone via the north entrance and via the mine assembly area is still marked.

The north entrance shortcut allows Sam to quickly move from the common area, where he begins the mission, to the low security corridor. This is essential given the expansion of the main restricted complex in this mission. But this new rhythm, where there are no quiet passages between the main area in the open access and restricted access areas, gives a more intense feel to this level when compared to the second.

In this level the main NSA mission is again on one of the most segregated lines. However, while the site of the task - the surveillance room, marked in Figure 9 with a yellow 
circle - is highly segregated in terms of access it also has many visual connections since its windows look directly down on the atrium around the general quarters and common area. If we weight connections according to visual connectivity, as suggested in Nicholas Sheep Dalton and Ruth Conroy Dalton's 'layered-graph' approach, we find that the surveillance room is very deep with respect to access but it is relatively shallow with respect to visibility [10]Error! Reference source not found. This discrepancy between the depth of the surveillance room in terms of access and in terms of vision is important to establishing its character and is a phenomenon that we will explore further later in the paper.

\section{Visual Graph Analysis}

\subsection{Enrica's workshop}

Figure 10 and Figure 11 show the site of one of the secondary NSA missions in level 3: Enrica's workshop. Here, Sam must enter by the door in the northwest, sneak past Enrica, who is sitting at the desk in the northeast with her back to the room, and get to the computer at the desk in the south. When he gets to the desk there is a trigger point that sends Enrica on the path marked in blue to the filing cabinet in the southeast corner. When she returns to her own desk Sam can hack into the computer, which is his task. He can then look in the filing cabinet to complete a secondary NSA mission.

On the right of Figure 11 is the same room under visual graph analysis showing isovist area. From each point in the room we can draw an isovist. These isovists are drawn from a crouching perspective, which is the position Sam adopts on entering the restricted area. Each isovist will have a particular area, which reflects the amount of space that can be seen from the isovist's starting point. The VGA graph colour codes this information, with large isovists shown in warm colours and smaller isovists shown in cold colours.

Figure 10: Sam enters Enrica's workshop and looks through files.

Figure 11: Enrica's workshop plan (left) and VGA showing isovist area (right). 
Enrica's position at her desk is in the middle of the most visually dominant area of the room. If she were to turn around she would be able to see a large part of the room. When Sam enters the room he is in the middle of a series of small isovists, thanks largely to the desk next to the door. An isovist of small area has two meanings in a game like Splinter Cell. On the one hand, it prevents the player from getting the lay of the land due to a restricted visual field. On the other, it hides the avatar from enemy patrols. The tension that is essential to stealth games is based on this trade-off.

Another source of tension in stealth games arises from the requirement for the player to continually put the avatar in harm's way. In the above example we can see that Sam must move from relatively safe positions (the blue areas close to the wall on the left) to more dangerous positions in the centre of the room in order to get to his goal. He must also occupy the orange position by the filing cabinet for the secondary task. Enrica stays in visually dominant positions throughout the task, going between the red and orange areas.

Thus the room is designed in such a way that a rhythm of tension and relief is established, first by forcing Sam to either move near Enrica or into a 'warm' area or both, and then by bringing Enrica close to Sam. The exact quality of these two forms of tension creation is different. The first form is due to the player being forced to act in a way that is risky. I do not want to go so close to Enrica or expose Sam in a large isovist but it is the only way to the goal. Here, player action is responsible for any anxiety induced by putting Sam in a vulnerable position. The second form is due to the player being forced to react to a threatening change in the environment. Now, the action - hiding under the desk - makes Sam safer, and allows for the resolution of tension. Of the two forms of tension-creation, the former is undoubtedly the more disturbing, since the ratcheting of the tension has its roots in the player rather than in an external agent.

\subsection{The low security corridor, third level}

The same pattern can be seen in a large space if we look at the low security corridor in the third level (Figure 12).

Figure 12: Isovist area for the low security corridor in the third level. 
In this level Sam enters the low security corridor through either of the doors circled in Figure 12. His goal is the surveillance room to the south. Whichever door he uses, Sam must move through the two red 'blotches' by the window that overlooks the atrium. Again, we have a situation where the player is forced to put Sam in harm's way in order to get to the goal. The difference between the two doors also shows up on the graph. From the east, Sam enters the low security corridor into a set of relatively small isovists. The player has time to spot the patrols and look for cover. If entering from the north, Sam comes straight into a set of fairly large isovists, which make for a more stressful entrance. As already noted, this northern door represents a shortcut between the complex's two sub-systems. The difference in the kinds of isovists that each entrance gives onto is part of how the game balances the benefits of this short-cut.

\section{Visibility/Accessibility Discrepancy (VAD)}

The discrepancy between the visibility and accessibility of the level's convex spaces are represented by the graphs in Figure 13 and Figure 14.

\section{Figure 13: VAD values for the first level. Warmer colours indicate higher values. 1: Long corridor. 2: Kitchen and sleeping area.}

At the start of the game, the highest values are found in the general quarters. Figure 13 shows the two areas in the general quarters with the highest values. These are 1) the long corridor and 2) the kitchen and sleeping area. A high VAD value means that these areas have visual connections to relatively inaccessible areas. In the case of the long corridor, this is due to a lot of visual connections to spaces that are fairly close (the back of the general quarters, the common area and the rooftop access) and a couple to areas that are distant (the low security corridor and the surveillance areas). In the case of the kitchen and sleeping area the high VAD value is largely due to the view of the courtyard, which is very distant. We can think of the general quarters as a balcony in Koch's sense. It allows the player to take in spaces that will be important during the game. Most important in this respect are the restricted areas that are viewable from the start of the game. The view from the kitchen and sleeping area onto the courtyard gives the player a glimpse of a currently inaccessible area that will become important 
in the second level, when the courtyard provides an alternative, hidden route to Emile's quarters. By including visual connections to the courtyard in the first level this hidden route is announced to the player, but in a relatively subtle way.

But these warm-coloured convex spaces around the general quarters may also be thought of as a catwalk. Visual connections to the surveillance area allow the player to see into the restricted area, but they also put Sam on display. This effect is particularly pronounced in the long corridor because the surveillance room is higher up than the general quarters, giving its occupants an advantage over Sam. This configuration establishes the theme of surveillance, emphasizing the feeling that Sam is continually observed.

As can be seen from the screenshot in Figure 7, the surveillance room is a part of the visual field for the player on entering the compound for the first time. However, it is on the upper periphery of the visual field, whereas the player's attention is generally directed horizontally and downward through the light sources from the TV and the corridor that leads to the training course, the direction of travel of the NPC Sam is instructed to follow, and the low roof of the balcony corridor, which partially obstructs the upper levels. This establishes the surveillance room, but does not give it an immediate importance in terms of access. Firstly, this registers it as a long-term goal in contrast to the short-term goals of the training course, the infirmary and the TV area, which are important spaces in the first mission. Secondly, it gives the surveillance room a more insidious presence than those rooms that are announced in a more straightforward way and occupy a more conscious part of the player's first impression of the compound. The surveillance room here demonstrates how 'weenies' of this kind can function not only as long or short term goals that structure motivation and orientation, but also as constant threat that establishes atmosphere.

In all three levels there is a clear distinction between the main area and the restricted area on the one hand and the lower ground floor and basement (the right hand complex on the plan, inside the yellow rectangle in Figure 13) on the other. This lower area, particularly as we get away from the common area, has low VAD values, giving the area a labyrinthine feel. The player generally only sees into directly accessible spaces rather than distant, inaccessible spaces. In areas with higher VAD values journeys can be planned in advance to a greater degree. In areas with lower VAD values the player is forced to make choices on the spot. That is, junctions and possible alternative routes are not announced in advance but only when the player arrives at 
them. The lower floor is associated throughout the game with particular horrors, and this sits well with the labyrinthine configuration. It is here that Sam is asked by the JBA leader to kill two innocent people, that Sam must dump a dead body, and that we find the executed body of Enrica. It is also here that we witness the explosion of a cruise ship. These events all serve to give this area of the complex a morbid atmosphere, but this atmosphere is already intimated by the area's configuration.

\section{Figure 14: VAD values for the third level. The two main rooms in the surveillance area are circled.}

In the third level the player can finally access the surveillance area, and this is the location of the main NSA goal in this level. We see here that the two main rooms in the surveillance area, both circled in Figure 14, have the largest discrepancy between visibility and accessibility; the largest VAD value. They each provide a venue which can see onto several areas but they are each relatively inaccessible, being at the end of a long series of spaces. Their high VAD score imbues these areas with a particular atmosphere that is evident throughout the game. This atmosphere is somewhere between the balcony and the glass box. As already mentioned, the surveillance room has an ambivalent presence in the player's visual field on entering the complex. It registers that Sam is being watched by the surveillance team on the balcony. But the surveillance room - closed off for the first two levels and then the deepest area in terms of access - remains an attractor. Of course, the player only gradually learns how deep the surveillance area is and only knows the full discrepancy once the room is attained. The VAD score is therefore revealed over the course of the game rather than being immediately obvious. There is a sense of triumph in getting to the room since it is signalled so far in advance. This visual foregrounding of a distant goal is seen in several games as a navigational aid but also as a means of stoking curiosity, maintaining the importance of the goal in the player's mind, and creating a sense of achievement once it is attained.

\section{Further research}

This paper has used space syntax and visibility analysis to discuss how configuration impacts on the navigability and atmosphere of a level in a stealth game. Future research could 
possibly investigate the feasibility of this type of analysis in other genres. VAD seems to play a part in puzzle games in particular, where the discrepancy between a highly visible but highly inaccessible goal is often a key pleasure in the genre. First person shooter maps rely a great deal on lines of sight and on the circulation paths through a level to control pace and offer different kinds of strategic challenges. Axial maps and VGA may prove useful in evaluating these kinds of maps.

Two problems with this approach might be noted with a view to further work in the area. Firstly, this approach contains certain assumptions that empirical work would help to clarify. For example, are these models (the convex map and the axial map in particular) meaningful in relation to game spaces? Much of the authority of space syntax as a method lies in the empirical work linking traffic and pedestrian flows to space syntactical measures. Games studies requires a body of similar work in game spaces. This would not only be useful in honing critical evaluations of game spaces but also in designing spaces in ways that can control player movement without seeming to hamper player freedom.

Secondly, Depthmap is a piece of software that models three-dimensional spaces in two dimensions for the purposes of analysis. While workarounds are possible to take account of the vertical dimension $[32,4]$ and attempts have been made at three-dimensional isovist analysis [21] and three-dimensional space syntax analysis [31], this remains a problem, particularly in larger spaces. The problem is particularly felt in analysing games, where the vertical dimension can be of particular importance. In the present study the visual graph analysis was restricted to a single floor in order to avoid this limitation of Depthmap, but if an analysis were to take on, for example, recent first person shooter multiplayer levels this would be an untenable approach.

\section{REFERENCES}

[1] E. Adams, The Role of Architecture in Video Games, Gamasutra, 2002.

[2] M. L. Benedikt, To take hold of space: isovist fields., Environment and Planning B: Planning and Design., 6 (1979), pp. 74-65.

[3] E. Bigand, R. Parncutt and F. Lerdahl, Perception of musical tension in short chord sequences: The influence of harmonic function, sensory dissonance, horizontal motion, and musical training., Perception \& Psychophysics, 58 (1996), pp. 125-141. 
[4] D. Chang and A. Penn, Integrated multilevel circulation in dense urban areas: the effect of multiple interacting constraints on the use of complex urban areas, Environment and Planning B: Planning and Design, 25 (1998), pp. 507-538.

[5] S. Chen and D. Brown, The Architecture of Level Design, Gamasutra, 2001.

[6] I. O. Cho and Y. O. Kim, The relationship between spatial configuration and spatial behavior in online game space., Proceedings Sixth International Space Syntax Symposium., Istanbul, 2007, pp. 1-14.

[7] Y. K. Choi, The Morphology of Exploration and Encounter in Museum Layouts, First International Space Syntax Symposium, 1997.

[8] S. Clarke-Wilson, Applying game design to virtual environments, in J. C. Dodsworth, ed., Digital Illusion: entertaining the future with high technology, ACM, New York, pp. 229-239.

[9] R. Conroy, Spatial Navigation in Immersive Virtual Environments, Bartlett School of Architecture, UCL, London, 2001.

[10] N. S. Dalton and R. C. Dalton, Solutions for visibility-accessibility and signage problems via layered-graphs, Journal of Space Syntax, 1 (2010).

[11] S. Girginkaya and G. Çağdaş, Comparison of movement models between real and virtual environments, Sixth International Space Syntax Symposium, 2007.

[12] B. Hillier, The Art of Place and the Science of Space, World Architecture, 185 (2005), pp. 96-102.

[13] B. Hillier, Space is the Machine. A configurational theory of architecture., University of Cambridge Press, Cambridge, 1996.

[14] B. Hillier and J. Hanson, The Social Logic of Space, Cambridge University Press, Cambridge, 1984.

[15] B. Hillier and A. Penn, Visible colleges: structures and randomness in the place of dicovery, Science in Context, 4 (1991), pp. 23-49.

[16] K. Hullett and J. Whitehead, Design Patterns in FPS Levels, Fifth International Conference on the Foundation of Digital Games, 2010.

[17] Infinity Ward, Call of Duty: Modern Warfare 2, Activision, 2009.

[18] D. Koch, Architecture re-configured, Journal of Space Syntax, 1 (2010).

[19] B. Kreimeier, The Case for Game Design Patterns, Gamasutra, 2002.

[20] R. M. Levy and M. G. O’Brien, A Virtual World for Teaching German, Loading ..., 1 (2007).

[21] E. Morello and C. Ratti, A Digital Image of the City: 3-D isovists in Lynch's urban analysis, Environment and Planning B: Planning and Design, 36 (2009), pp. 837-853.

[22] M. Nitsche, Video Game Spaces. Image, play and structure in 3D worlds, The MIT Press London, 2008.

[23] Number None, Braid, Microsoft Game Studios, 2008.

[24] N. Orellana, On Spatial Wayfinding: Agent and Human Navigation Patterns in Virtual and Real Worlds., Bartlett School of Graduate Studies, UCL, London, 2012.

[25] A. Penn, Space syntax and spatial cognition. Or, why the axial line?, Proceedings of the 3rd International Space Syntax Symposium, Atlanta, 2001.

[26] J. Peponis, The spatial culture of factories, Human Relations, 38 (1985), pp. 357-90.

[27] J. Peponis, C. Zimring and Y. K. Choi, Finding the Building in Wayfinding, Environment and Behavior, 22 (1990), pp. 555-590.

[28] Playdead, Limbo, Microsoft Game Studios, 2010. 
[29] S. Psarra, Architecture and Narrative: The formation of space and cultural meaning in buildings, Routledge, Abingdon, 2009.

[30] S. Rogers, Everything I Learned about Game Design I Learned from Disneyland, Mr. Boss' Design Lair, 2009.

[31] C. Schroder, W. Mackaness and F. Reitsma, Quantifying Urban Visibility Using 3-D Space Syntax, GISRUK 2007, 2007.

[32] A. Turner, Depthmap 4: A Researcher's Handbook, UCL, London, 2004.

[33] Ubisoft Shanghai, Splinter Cell: Double Agent, Ubisoft, 2006.

[34] Valve Corporation, Portal, Valve Corporation, 2007. 